\title{
Solubility analysis of venlafaxine hydrochloride polymorphs by shake-flask method and real time monitoring
}

\author{
KRISZTINA TAKÁCS-NOVÁK ${ }^{1 *}$, DÓRA TEMPFLI ${ }^{1}$, DÓRA CSICSÁK ${ }^{1}$, GERGELY VÖLGYI ${ }^{1}$, \\ ENIKŐ BORBÁS², ZSOMBOR K. NAGY², BÁLINT SINKÓ3
}

\author{
${ }^{1}$ Department of Pharmaceutical Chemistry, Semmelweis University, H-1092 Budapest, Hőgyes Endre u. 9., Hungary \\ ${ }^{2}$ Department of Organic Chemistry and Technology, Budapest University of Technology and Economics, \\ H-1111, Budapest, Müegyetem rakpart 3., Hungary \\ ${ }^{3}$ Pion Inc. Billerica, Massachusetts 01821, United States of America
}

*Corresponding author: Krisztina Takács-Novák

Email: novak.krisztina@pharma.semmelweis-univ.hu

Received: 21 May 2019 / Accepted: 2 July 2019 / Published online: 24 July 2019

\begin{abstract}
Aims: The aqueous solubility of two polymorphic forms of venlafaxine hydrochloride was investigated.

Methods: The $\mathrm{pH}$-dependent solubility $\left(S_{p H}\right)$ over a wide $\mathrm{pH}$ range was measured by saturation shake-flask (SSF) method at $25^{\circ} \mathrm{C}$. The solubility of the free base form was depicted by the intrinsic solubility $\left(S_{0}\right)$. To identify the solid form present at the solubility equilibrium, X-ray powder diffraction (XRPD) and Raman spectroscopy was carried out. The dissolution was studied using real time concentration monitoring applying fiber optic UV probes.

Results: No difference was found in the $S_{p H}$ values of Form I and Form II, in the $p H$ range 7.5-12. Solid phase isolated from $p H$ 10-12 suspensions was identified as free base by XRPD and Raman spectroscopy. Precipitates separated from $\mathrm{pH}$ 7-8 samples were also identical product. The transition of polymorphs to the free base was supported by the real time dissolution analysis.

Conclusion: In this study we demonstrated a good agreement of equilibrium solubility measured by SSF method and in-situ UV fiber optic method. $\mu$ DISS Profiler ${ }^{T M}$ has the advantage to provide much more information about dissolution process; with this approach the dissolution kinetic, the supersaturation and the time needed to reach the equilibrium can be easily monitored.
\end{abstract}

Keywords: solubility-pH profile, polymorphs, shake-flask method, $\mu$ DISS Profiler, venlafaxine

\section{Introduction}

Solubility is one of the most important molecular properties of drugs. It plays a key role in determining their absorption potential and has fundamental impact on the bioavailability therefore. Solubility data refers to the given physical form of a solid compound thereby it is significantly affected by polymorphism. Polymorphism - the ability of a substance to exist as two or more crystalline forms that have different arrangements and/or conformations of the molecules in the crystal lattice - is common among the pharmaceutical solids [1]. More than one third of drugs show polymorphism and a further one third is capable of forming hydrates and solvates [2]. This subject has gained intensive attention in both original and generic drug research since in case of several drugs polymorphism caused serious safety problems [3]. The most known examples (from chloramphenicol palmitate, through carbamazepine, oxytetracycline, enalapril, etc. up to ritonavir) have been extensively reported in the literature [4-8]. These cases in- duced severe regulatory considerations [9] resulting that polymorph screening and the investigation of physico-chemical properties and stability of different polymorphic forms has become inevitable during the process of drug development [10, 11].

Polymorphs have different lattice energy so they differ in those physical and chemical properties which associated with the internal free energy of the solid, such as: density, refractivity, conductivity, hygroscopicity (packing properties); melting temperature, vapor pressure, solubility (thermodynamic properties); dissolution rate, stability (kinetic properties); and several others (surface, mechanical, spectroscopic properties) [2]. From absorption and bioavailability point of view the solubility and the dissolution rate are the most relevant properties of a pharmaceutical compound, however from formulation and safety aspects stability is also essential. A general rule is that a polymorph having the lower lattice free energy is the more stable form and it has the lower solubility while that having higher energy is less stable but tends to dissolve faster and has higher solubility [1]. Based on litera- 
ture solubility data Pudipeddi and Serajuddin [12] published a survey that the ratio of metastable/ stable polymorph solubility is typically less than 2 , though occasionally higher ratios can be observed as reported for sulindac ( $7 x$ difference between From I and II) [13] and for premafloxacin (23x) [14], etc. Similar trend was found for anhydrate/hydrate solubility ratio $(\sim 2)$ with many exceptions, while the amorphous/crystalline forms solubility differs with factor $\sim 10$, generally. Solubility difference of polymorphs will have effect on the bioavailability/bioequivalence $(\mathrm{BA} / \mathrm{BE})$ of a drug product if the solubility is the rate limiting factor upon absorption (for BCS II and IV drugs).

Solubility characterized by the equilibrium solubility data is the concentration of the compound in a saturated solution when solid and solute are at equilibrium. This parameter can be measured by several methods however the "gold standard" is still the saturation shake-flask technique (SSF). Recently Avdeef et al. published a consensusbased "white paper" commentary which summarized the recommendations for solubility measurement including SSF and other methods to improve the data quality [15]. The paper pointed out to the difficulties in the measurement of polymorphs due to the possible polymorph transitions upon equilibration and the importance of the analysis of both solution and solid phases. Well established methods for solid phase analysis (X-ray powder diffraction XRPD, differential scanning calorimetry DSC, FT-IR and Raman spectroscopy, etc.) should be used for characterization of the solid at the beginning and also at the end of the solubility experiment. Adhering and applying all these recommendations of the "white paper" one can measure only the thermodynamic solubility of that crystalline form which is in dynamic equilibrium with its solution form, at the temperature of the experiment. However, during the dissolution process polymorphs can show different kinetics, resulting in substantially various supersaturation solutions even so if later they convert to another form. The incubation time in the standard protocol of SSF is $24 \mathrm{~h}$ (6 h agitation and $18 \mathrm{~h}$ sedimentation) [16]. Since solvent induced changes in solid form structure might occur much faster or slower than this timeframe the in situ monitoring of the dissolution-time profile may provide more information about the behavior of polymorphs and may enable the optimization of incubation time. Moreover in situ monitoring with fiber optic UV probes under certain con- ditions (low amount of excess solid, long analysis time, etc) enables the determination of dissolution kinetics and also equilibrium solubility from a single experiment.

The goals of the present study were: (1) investigation of equilibrium solubility of different polymorphs of venlafaxine hydrochloride (VENL) as model compound, in wide $\mathrm{pH}$ range by SSF method coupled with solid phase analysis; (2) real time monitoring of the dissolution profile using in situ UV fiber optic; (3) to compare the equilibrium solubility results obtained by SSF and $\mu$ DISS methods and (4) to reveal the advantages and limits of the latter technique for solubility measurement.

\section{Material and methods}

\subsection{Materials}

The structure of model compound is presented in Figure 1. Venlafaxine hydrochloride $(313.86 \mathrm{~g} / \mathrm{mol})$ was purchased from Sigma-Aldrich Co. LLC. (St. Louis, MO, USA). From the commercially available polymorph the other polymorphic form was prepared in house based on patent (US6924393B2) and verified by XRPD. Distilled water of Ph. Eur. grade was used. All other reagents were of analytical grade.

A Britton-Robinson (BR) buffer stock solution (a mixture of acetic acid, phosphoric acid and boric acid, each at $0.04 \mathrm{M}$ ) was prepared, and the required amount of $0.2 \mathrm{M} \mathrm{NaOH}$ or $1 \mathrm{M} \mathrm{NaOH}$ was added to give the $\mathrm{pH}$ specified for the solubility experiments. BR buffer solutions were used in $\mathrm{pH}$ range 7.5-11, while at $\mathrm{pH} 11$ and above $\mathrm{NaOH}$ was applied.

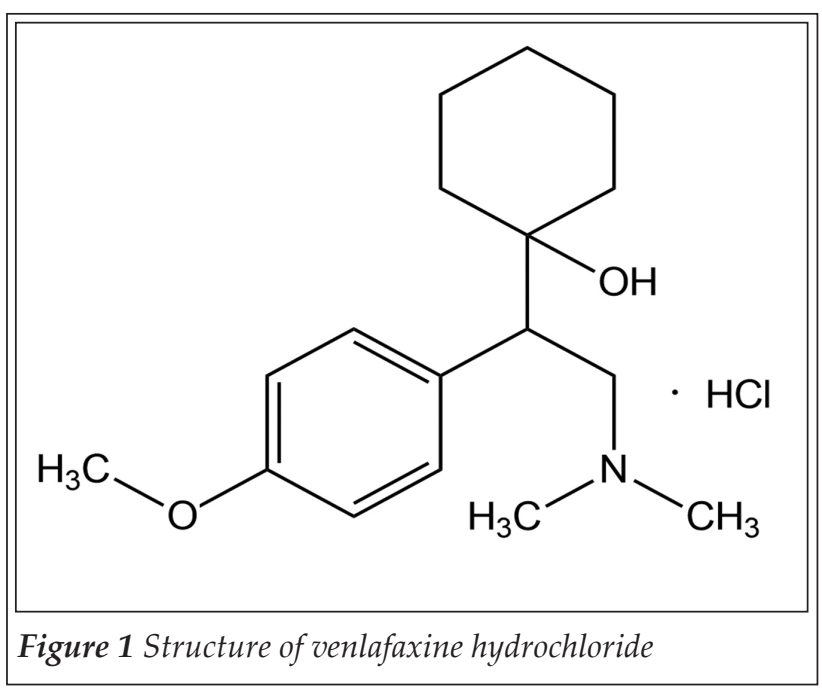




\subsection{Determination of the thermodynamic solubility by saturation shake-flask method (SSF)}

\subsubsection{Preparation of saturated solution}

The equilibrium solubility of VENL in the examined $\mathrm{pH}$ region was determined by the SSF method $[16,17]$. The sample was added in excess to the aqueous buffer solutions to produce a suspension. The amount of solid added was accurately measured: $10-300 \mathrm{mg} / 4 \mathrm{~mL}$. At controlled temperature of $25.0 \pm 0.1{ }^{\circ} \mathrm{C}$ the solution containing solid excess of the sample was vigorously stirred for a period of $6 \mathrm{~h}$ (agitation time), what followed a further $18 \mathrm{~h}$ of sedimentation period (stirrer turned off).

\subsubsection{Concentration determination of the saturated solution}

\subsubsection{Off-line: sampling, dilution and concentration measurement with UV spectrophotometer}

The concentration of the saturated solution was measured by UV spectroscopy using JASCO V 550 UV/VIS spectrophotometer. Three aliquots were carefully withdrawn from the liquid, using a fine glass pipette, and were diluted with the solvent if necessary. Three replicate solubility measurements were carried out at each of the tested conditions.

The specific absorbance $\left(\mathrm{A}^{1 \%}{ }_{1 \mathrm{~cm}}\right.$ the absorbance of $1 \mathrm{~g} / 100 \mathrm{~mL}$ solution over a $1 \mathrm{~cm}$ optical pathlength at a given wavelength) of VENL was determined separately at $\mathrm{pH} 8.0$ using 12 points of two parallel dilution series, from the linear regression equation (Lambert-Beer law) where the regression coefficient ( $\mathrm{r}$ ) was higher than 0.9998 . The specific absorbance data obtained is 38.4 at $\lambda_{\max } 273.5 \mathrm{~nm}$. This value was used for concentration calculation at all $\mathrm{pHs}$ since the UV spectrum of VENL is not $\mathrm{pH}$-dependent due to the distance between the protonation site (aliphatic $\mathrm{N}$ atom) and the chromophore in the structure.

\subsubsection{In situ: with fiber optic UV probe} $\mu$ DISS Profiler ${ }^{\mathrm{TM}}$ (Pion Inc. Billerica MA, US, Figure 2) has been applied as UV fiber optic instrumentation. The equilibrium concentration was concomitantly determined in situ in the equilibrated solubility suspension (without dilution) immersing the UV dip probe. Calibration was performed with the same $(2-5-10-20 \mathrm{~mm})$ tip adjusted to the fiber optic UV dip probe which was used in the given

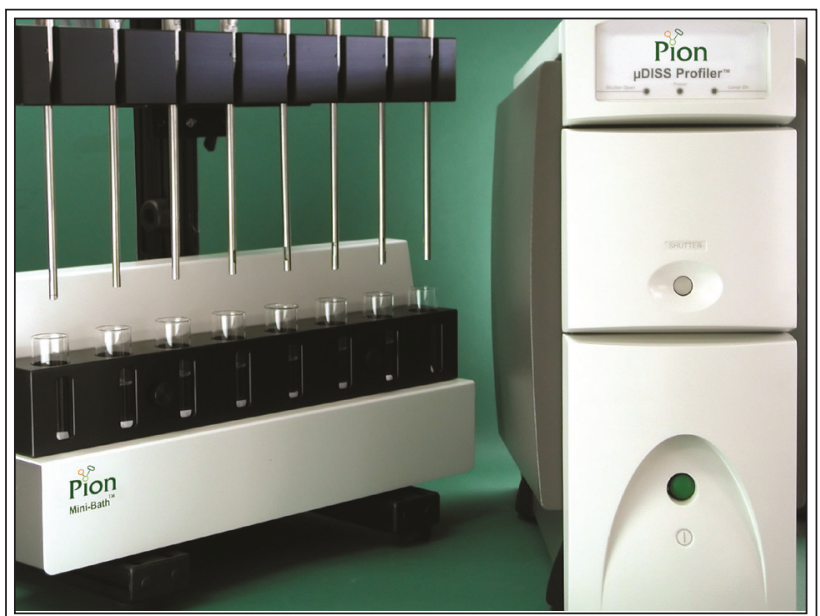

Figure $2 \mu$ DISS Profiler ${ }^{T M}$

solubility experiment. Solubility values obtained from the same experiments analysed with both the off-line and the in situ UV method were compared (see section 3.3.).

\subsection{In situ real time monitoring of dissolution, supersaturation, precipitation and the equilibrium solubility}

The drug solution concentration versus time (0-24 h) was investigated with the $\mu$ DISS Profiler ${ }^{\mathrm{TM}}$. The instrument measures the real time concentration with fiber optic UV dip probe inserted in 6 temperature controlled $20 \mathrm{~mL}$ vessels, stirred with magnetic stirrer. Dissolution of VENL was registered in BR buffer $\mathrm{pH} \sim 11.5$, in three parallel channels, where $10 \mathrm{mg}$ sample was added to $4 \mathrm{~mL}$ dissolution media. Using the appropriate $2 \mathrm{~mm}$ tip adjusted to the UV probe, the UV spectra were registered in the following timing protocol: per $1 \mathrm{~min}$ in $0-2 \mathrm{~h}$, per $10 \mathrm{~min}$ in $2-6 \mathrm{~h}$ and per $30 \mathrm{~min}$ in 6-24 h periods. The stirrer was turned off at $6 \mathrm{~h}$. For the evaluation of the concentration previously determined calibration data and second derivative spectra were used.

\subsection{Methods for the analysis of the solid phase}

\subsubsection{XRPD}

Small amount of the solid phase at the end of solubility measurement was isolated and dried to a glass plate. X-ray powder diffraction patterns of these samples were recorded by means of a PANalytical (Amelo, The Netherlands) X'pert ProMDP X-ray diffractometer using $\mathrm{Cu}-\mathrm{K}^{\prime \prime} \alpha$ radiation $(1.524 \AA$ Á) and a Ni filter. The applied voltage 
was $40 \mathrm{kV}$, while the current was $30 \mathrm{~mA}$. The samples were analysed between $4^{\circ}$ and $42^{\circ} 2 \theta$.

\subsubsection{Raman spectroscopy}

Raman spectra were collected using a Horiba JobinYvon LabRAM system coupled with an Olympus 97 BX-40 optical microscope (Olympus Corporation, Tokyo, Japan). The sample was illuminated by a 785-nm diode laser (TEC 510 type, Sacher Lasertechnik, Marburg, Germany) and an objective of $\times 10$ (laser spot size, approximately $4 \mu \mathrm{m}$ ) was used for optical imaging and spectrum acquisition. The laser beam is focused through the objective, and the backscattered radiation is collected with the same objective, a common configuration found in most confocal spectroscopic systems. The collected radiation is directed through an edge filter that removes the Rayleigh photons and then through a confocal hole $(500 \mu \mathrm{m})$ and the entrance slit $(100 \mu \mathrm{m})$. Finally, a 950 groove/ $\mathrm{mm}$ grating monochromator disperses the Raman photons before reaching the CCD detector. Each spectrum of the image was collected in the spectral range of 300 to $1600 \mathrm{~cm}^{-1}$.

\subsubsection{Polarized light microscopy}

Polarized light microscopic images were taken using Amplival (Carl Zeiss, Jena, Germany) type microscope coupled with Olympus C4040 Z type camera (Olympus, Japan).

\subsection{Statistical analyses}

Concentrations were expressed as means \pm SD, and were compared using "two-sample" Student's $t$ test. Differences were considered statistically significant when $\mathrm{p}<0.05$.

\section{Results and Discussion}

In this study we measured the equilibrium solubility of different polymorphic forms of venlafaxine hydrochloride modelling the possible behaviour of a pharmaceutical solid. The $\mathrm{pH}$-dependent equilibrium solubility $\left(\mathrm{S}_{\mathrm{pH}}\right)$ over a wide $\mathrm{pH}$ range was investigated by SSF method at $25^{\circ} \mathrm{C}$ temperature. The solubility of the free base form was depicted by the intrinsic solubility $\left(\mathrm{S}_{\mathrm{o}}\right)$ measured at high $\mathrm{pH}$ value. To identify the solid form that is present at the solubility equilibrium, the approach was completed with the solid phase analysis at the end of the solubility experiment. We also studied the dissolution kinetics and the extent of supersaturation and the incubation time needed to reach the equilibrium, using real time monitoring by $\mu$ DISS Profiler ${ }^{\mathrm{TM}}$ to reveal the differences between the stable and less stable/metastable forms.

Venlafaxine is a $2^{\text {nd }}$ generation, SNRI (serotoninnorepinephrine reuptake inhibitor) antidepressant drug nowadays widely used in depression, in generalized anxiety disorder, post-traumatic stress disorder, etc. (US Patent, 2006, US7030164B2). VENL is used as racemate of its hydrochloride salt which is known to exist in two polymorphic forms, Form I and Form II. Though Form I is more stable (melting temperature: $210-212{ }^{\circ} \mathrm{C}, \Delta \mathrm{H}: 125.8 \mathrm{~J} / \mathrm{g}$ ) than Form II (melting temperature: 208-210 ${ }^{\circ} \mathrm{C}$, $\Delta \mathrm{H}: 130.3 \mathrm{~J} / \mathrm{g}$ ) the latter is preferred in the formulation because it has larger particle size with better filtration and drying characteristics [18]. VENL is a monovalent base $\left(\mathrm{p} K_{a}: 9.63\right)$, its solubility is $\mathrm{pH}$ dependent. Since the compound belongs to BCS I class, the solubility is not an absorption limiting factor from GI tract. Investigation of salt solubility in biorelevant medium was not the goal of this study. However VENL is a good model for studying the $\mathrm{pH}$-dependent solubility of polymorphs and for comparison the SSF and $\mu$ DISS methods in the measurement of intrinsic solubility.

Table I Equilibrium solubility of venlafaxine hydrochloride polymorphs in BR buffer measured by SSF method

\begin{tabular}{c|c|c|c|c|c}
\hline \multicolumn{3}{|c|}{ VENL Form I } & \multicolumn{3}{c}{ VENL Form II } \\
\hline $\mathbf{p H}$ & $\begin{array}{c}\mathbf{S}_{\mathbf{p H}} \pm \mathrm{SD} \\
(\mathbf{m g} / \mathbf{m L})\end{array}$ & $\mathbf{n}$ & $\mathbf{p H}$ & $\begin{array}{c}\mathbf{S}_{\mathbf{p H}} \pm \mathrm{SD} \\
(\mathbf{m g} / \mathbf{m L})\end{array}$ & $\mathbf{n}$ \\
\hline 7.50 & $59.35 \pm 8.23$ & 9 & 7.54 & $54.32 \pm 4.43$ & 3 \\
\hline 7.70 & $32.08 \pm 2.60$ & 9 & 7.60 & $45.67 \pm 2.34$ & 9 \\
\hline 7.83 & $23.34 \pm 0.45$ & 3 & 7.67 & $38.35 \pm 2.99$ & 9 \\
\hline 8.30 & $8.655 \pm 0.267$ & 9 & 7.78 & $26.79 \pm 0.27$ & 3 \\
\hline 8.34 & $7.194 \pm 0.043$ & 3 & 8.20 & $8.175 \pm 1.061$ & 9 \\
\hline 8.37 & $7.495 \pm 0.601$ & 3 & 8.40 & $7.689 \pm 0.345$ & 9 \\
\hline 8.43 & $6.983 \pm 0.103$ & 3 & 8.52 & $5.506 \pm 0.104$ & 3 \\
\hline 8.47 & $6.364 \pm 0.071$ & 3 & 8.58 & $4.985 \pm 0.053$ & 3 \\
\hline 9.30 & $0.948 \pm 0.031$ & 9 & 8.64 & $4.587 \pm 0.013$ & 3 \\
\hline 9.50 & $0.796 \pm 0.094$ & 9 & 8.70 & $3.809 \pm 0.104$ & 3 \\
\hline 9.72 & $0.574 \pm 0.011$ & 3 & 9.45 & $0.831 \pm 0.027$ & 9 \\
\hline 9.76 & $0.507 \pm 0.004$ & 3 & 9.76 & $0.567 \pm 0.014$ & 4 \\
\hline 9.81 & $0.559 \pm 0.062$ & 3 & 9.96 & $0.544 \pm 0.011$ & 3 \\
\hline 9.96 & $0.570 \pm 0.013$ & 3 & 10.06 & $0.495 \pm 0.052$ & 4 \\
\hline 10.34 & $0.521 \pm 0.186$ & 3 & 10.26 & $0.523 \pm 0.037$ & 4 \\
\hline 10.41 & $0.454 \pm 0.011$ & 3 & 10.41 & $0.498 \pm 0.034$ & 4 \\
\hline 11.51 & $0.275 \pm 0.004$ & 4 & 11.62 & $0.296 \pm 0.025$ & 4 \\
\hline 12.11 & $0.292 \pm 0.007$ & 4 & 12.01 & $0.285 \pm 0.004$ & 4 \\
\hline 609 & & & & 4 \\
\hline
\end{tabular}

Concentration is expressed as free base equivalents. 


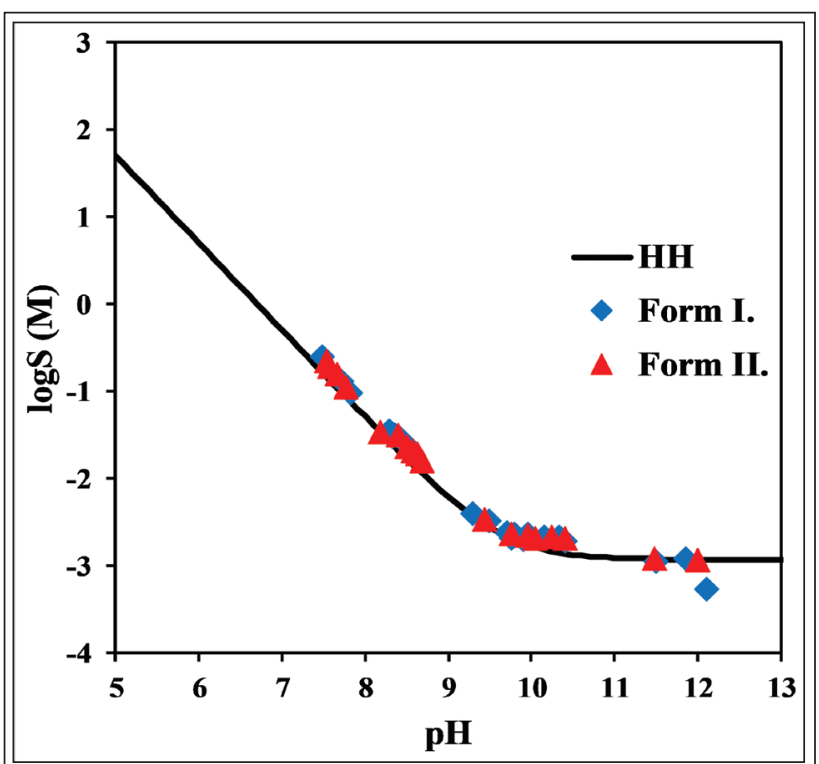

Figure 3 Solubility-pH profile of VENL Form I and Form II (solid line represents the theoretical HH curve calculated from $p K a: 9.63$ and $\log S o:-2.93$, points represent the experimental data)

\subsection{Solubility-pH profile of venlafaxine polymorphs}

The equilibrium solubility of VENL Form I and II were measured by the standard protocol of SSF method at 18 different points in $\mathrm{pH}$ range $7.5-12$, at $25^{\circ} \mathrm{C}$. The $\mathrm{S}_{\mathrm{pH}} \pm \mathrm{SD}$ results are expressed in $\mathrm{mg} / \mathrm{mL}$ unit as average value of parallel measurements and are presented in Table I. The standard deviation was in the range of $1-13 \%$, average SD: $6 \%$. No significant difference was found between the $S_{p H}$ values of polymorphs at the same $\mathrm{pH}$ in the whole exam- ined region. This can be interpreted with the transition of polymorphs to a common product as supported by the solid phase analysis (see below 3.2).

Figure 3 shows the solubility-pH profile of VENL Form I and Form II as plotted logS [M] values $v s \mathrm{pH}$. As it can be seen in Figure 3 the curve is typical for a monoprotic base as described by the theoretical Henderson-Hasselbalch $(\mathrm{HH})$ relationship $\left(\log S=\log S_{0}+\log \left(10^{\mathrm{pKa}-\mathrm{pH}}+1\right)[17,19]\right.$.

The intrinsic solubility of VENL base (calculated as the average of values measured at $\mathrm{pH} \geq 11.5$ ) is $\log S_{0}[M]:-2.93 \pm 0.014(n=4)$. This value was used to generate the HH curve of VENL (solid line on Figure 3). The experimental data points closely follow the theoretical $\mathrm{HH}$ curve. Below $\mathrm{pH} 10$ the VENL free base starts to convert to cationic form and the solubility is increasing. Below $\mathrm{pH} 7$ the solubility was not studied because the salt solubility is very high $(>500 \mathrm{mg} / \mathrm{mL})$ and its saturated solution can be considered non-ideal solution.

\subsection{Characterization of the solid phase isolated from solubility experiment}

First the starting materials, Form I and Form II were identified by powder X-ray diffraction (see in Figure 4). Next the samples isolated and dried from the solubility suspension after incubation at different $\mathrm{pHs}$ were studied. Diffractograms shown representatively in increasing $\mathrm{pH}$ order in Figure 4 demonstrate that the samples are not identical with the starting polymorphic forms in neither case. Results indicate that both polymorphs re-


Figure 4 XRPD patterns of VENL Form I and Form II and the solids isolated from solubility suspensions at different $\mathrm{pH}$ values 


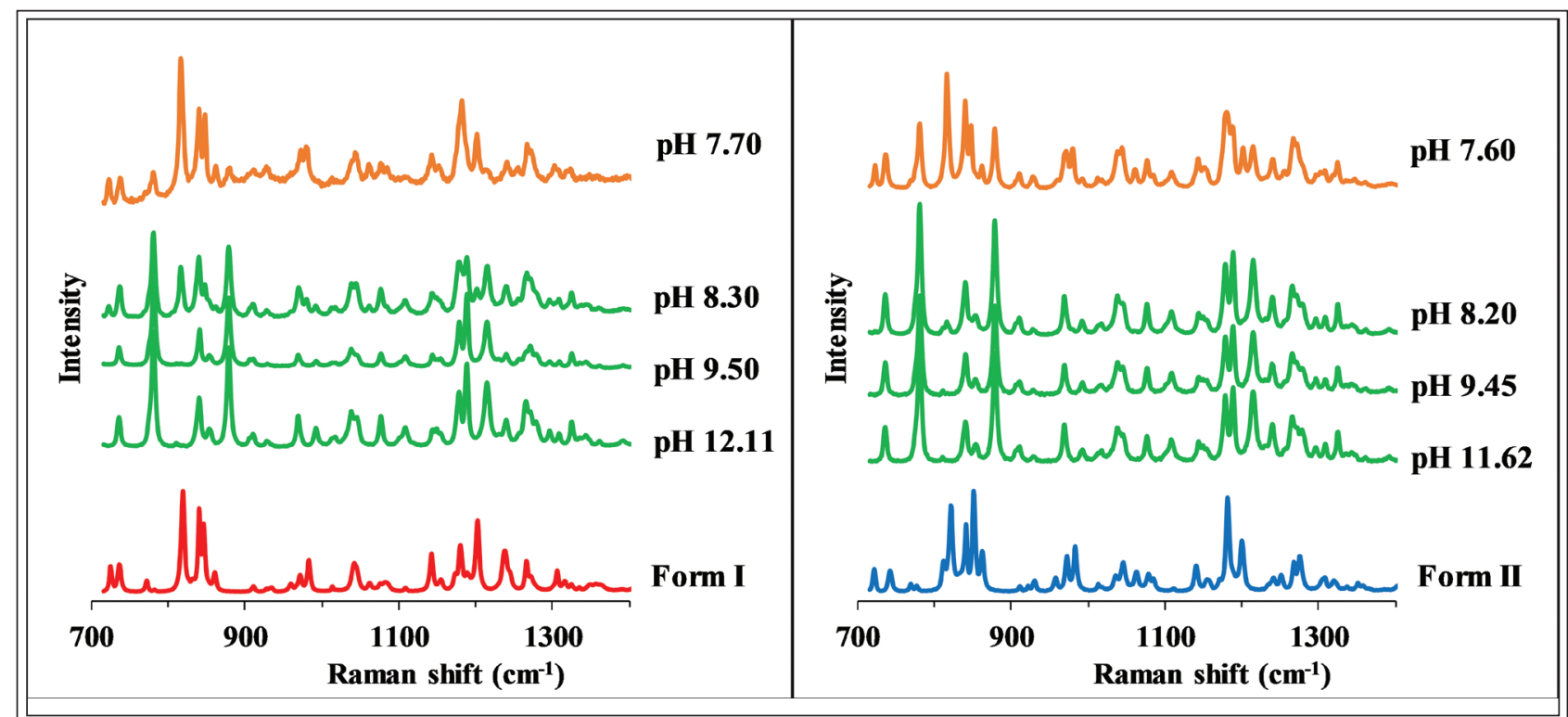

Figure 5 Raman spectra of solid phase of VENL polymorphs isolated from solubility suspensions

crystallized to a common form. Product obtained at lower pHs (7.5-9) could not be identified using Cambridge Structural Database. Based on patent US20030114536A1 we identified the product as venlafaxine hydrochloride monohydrate. Patterns registered at higher $\mathrm{pH}$ (11-12) values were found identical with one of the free base VENL polymorphs, specified in the database as free base Form I. A peak (if any) appeared with various intensity in the patterns of free base at 9 ( 2 Theta) is due to the presence of sodium acetate remained in some samples. XRPD finding was supported by Raman spectroscopy as well (Figure 5). The polarized light microscopic images in Figure 6 (A and B panels) indicate that crystal shape of Form I and II is column but they substantially differ in their
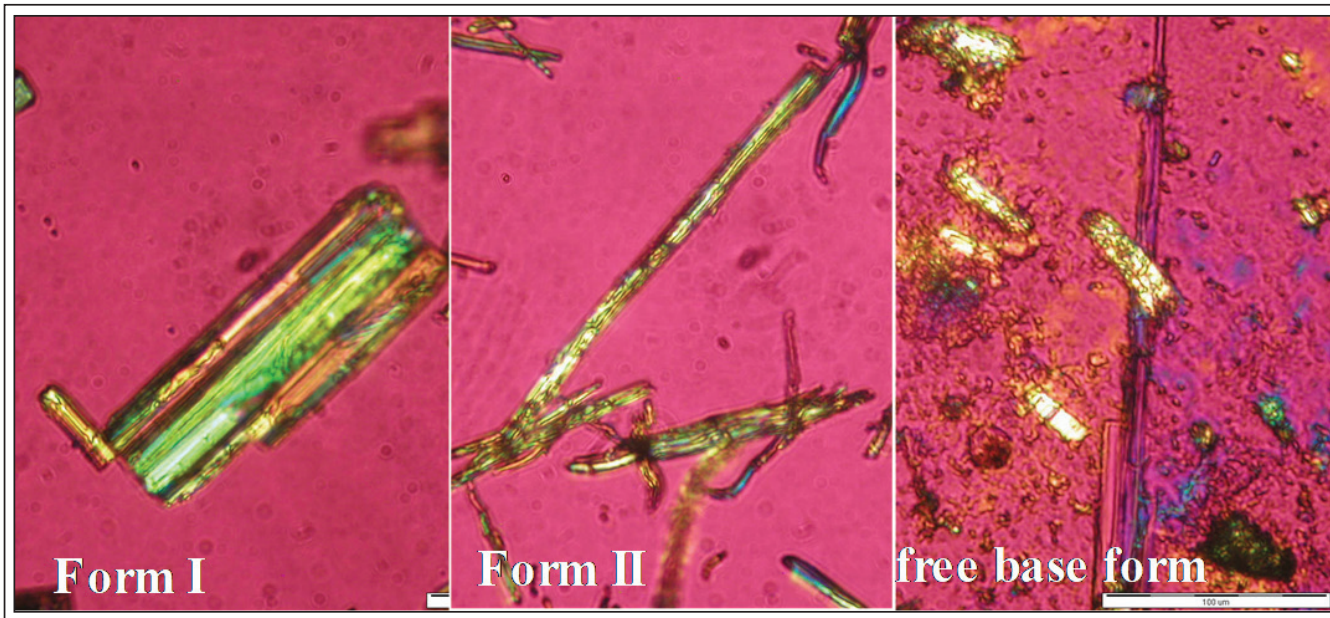

Figure 6 Polar microscopic images of VENL samples (A: Form I, B: Form II, C: free base isolated at pH 12) size. Panel $\mathrm{C}$ shows the shape of the free base form isolated at $\mathrm{pH} 12$.

All above results of solid phase analysis confirm and can interpret the equilibrium solubility data measured experimentally (Table $I$ ) in $\mathrm{pH}$ range 7.5-12.

\subsection{Comparison of concentration measurement with off-line UV method and in situ UV probe}

$\mu$ DISS Profiler ${ }^{\mathrm{TM}}$ measures the concentration of the solute in situ in the saturated solution in the presence of solid. In order to prove that it has no impact on the results (e.g. distorting the absorption measurement), in a part of SSF experiment at the end of incubation time the concentration of the 

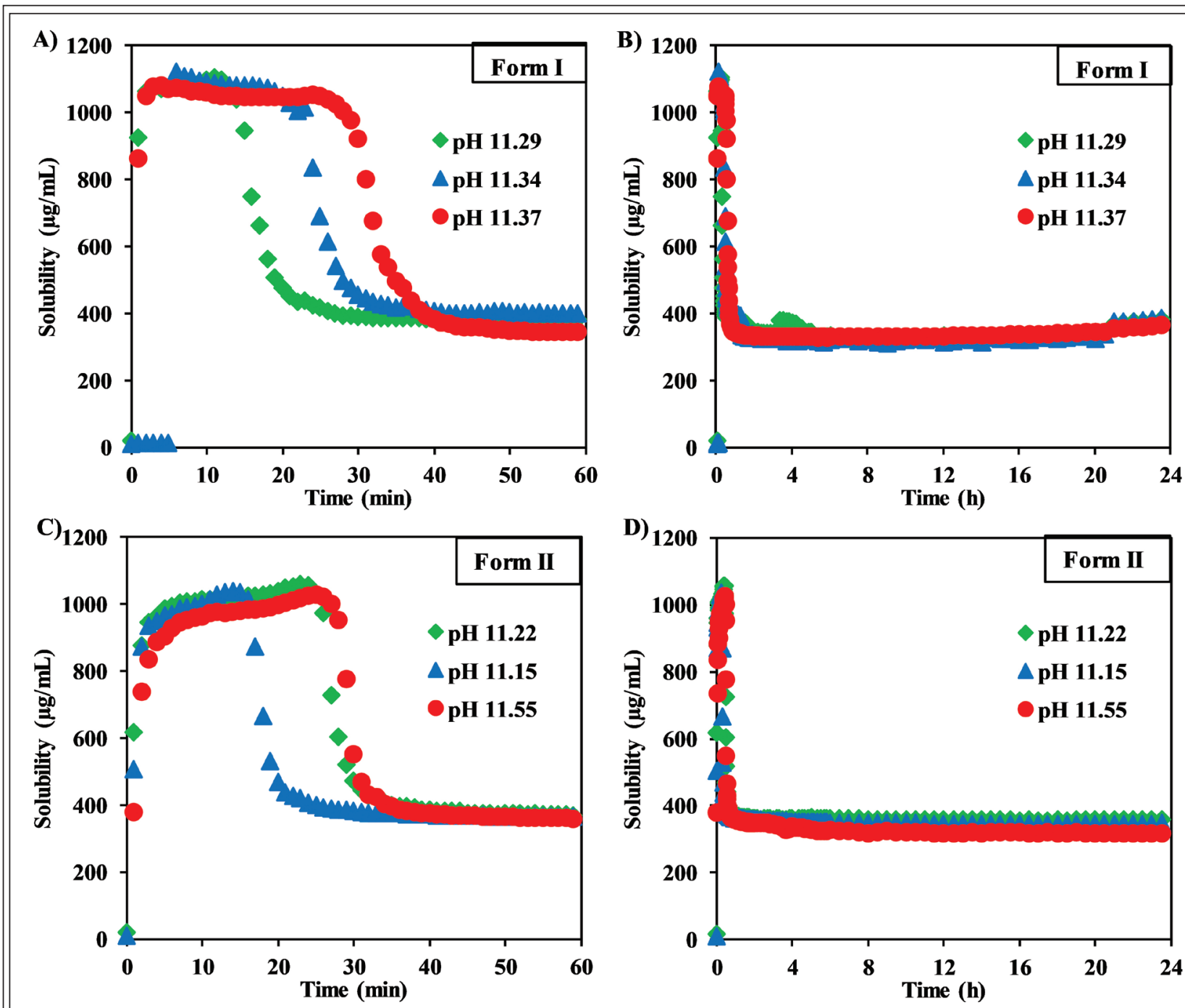

Figure 7 Solubility-time profile of VENL Form I (upper panels) and Form II (lower panels) at pH 11.5 in BR buffer monitored by $\mu$ DISS approach

Table II Equilibrium solubility of venlafaxine hydrochloride polymorphs measured by SSF method using different concentration detection

\begin{tabular}{|c|c|c|c|}
\hline \multirow{2}{*}{$\begin{array}{l}\text { Sample } \\
\text { VENL }\end{array}$} & \multirow[b]{2}{*}{$\mathrm{pH}$} & \multicolumn{2}{|c|}{$\mathrm{S}_{\mathrm{pH}} \pm \mathrm{SD}(\mathrm{mg} / \mathrm{mL})$} \\
\hline & & UV-JASCO & UV- $\mu$ DISS \\
\hline \multirow{5}{*}{ Form I } & 7.50 & $59.35 \pm 8.23$ & $56.98 \pm 6.89$ \\
\hline & 7.70 & $32.08 \pm 2.60$ & $31.34 \pm 2.40$ \\
\hline & 7.83 & $23.34 \pm 0.45$ & $22.44 \pm 0.31$ \\
\hline & 9.30 & $0.948 \pm 0.031$ & $1.121 \pm 0.038$ \\
\hline & 9.50 & $0.796 \pm 0.094$ & $0.764 \pm 0.080$ \\
\hline \multirow{5}{*}{ Form II } & 7.54 & $54.32 \pm 4.43$ & $48.51 \pm 6.32$ \\
\hline & 7.60 & $45.67 \pm 2.34$ & $45.54 \pm 1.25$ \\
\hline & 7.67 & $38.35 \pm 2.99$ & $36.49 \pm 0.45$ \\
\hline & 7.78 & $26.79 \pm 0.27$ & $26.96 \pm 0.40$ \\
\hline & 9.45 & $0.831 \pm 0.027$ & $0.853 \pm 0.030$ \\
\hline
\end{tabular}

Concentration is expressed as free base equivalents. varies in $0.2-15 \%$, average is about $5 \%$. It can be observed that in $7 / 10$ cases $\mu$ DISS measures the lower value.

\subsection{Real time monitoring of dissolution by $\mu$ DISS Profiler $^{T M}$}

The real time dissolution profile was monitored by $\mu$ DISS Profiler ${ }^{\mathrm{TM}}$. It was performed in BR buffer $\mathrm{pH} \sim 11-11.5$ (exact $\mathrm{pH}$ was measured at the end of the experiments) in $0-24 \mathrm{~h}$ period, where in the first $6 \mathrm{~h}$ stirrer was turned on. The UV spectra were registered according to a protocol (see in section 2.3.) in three parallel vessels and the concentration was calculated using calibration. Figure 7 shows that dissolution process of VENL can be followed well and the equilibrium solubility can 
also be measured by this approach. In case of Form I (Figure 7 upper panels) the supersaturation is very similar in the three parallel experiments and solute reaches 3.5 times concentration relative to the equilibrium solubility. Precipitation starts at different time in the parallels (12-25-30 $\mathrm{min}$, respectively) and the samples are reaching the equilibrium in rather short time (20-40 $\mathrm{min})$. The concentration is not changing further when at $6 \mathrm{~h}$ stirrer is turned off up to $20 \mathrm{~h}$. A small ( 13\%) increase in concentration can be observed in 20-24 $\mathrm{h}$ period for one of the channels. It may be due to the adsorption of solid crystals to the UV probe without stirring of the suspension. The dissolution-time profile of Form II as starting material (Figure 7 lower panels) is very similar. It reaches a bit slower the same supersaturation as Form I but the precipitation and time needed to reach the equilibrium is the same. We can conclude from this profiling that VENL polymorphs are converting to the common free base form immediately after dissolution. Intrinsic solubility measured by $\mu$ DISS Profiler ${ }^{\mathrm{TM}}$ at $24 \mathrm{~h}$ is $0.290 \pm 0.004 \mathrm{mg} / \mathrm{mL}$ $\left(\log S_{\mathrm{o}}[\mathrm{M}]:-2.98\right)(\mathrm{n}=4)$, it perfectly agrees with $\mathrm{S}_{\mathrm{o}}$ obtained by SSF method.

\section{Conclusions}

In this study we have demonstrated the importance of solid phase analysis in the solubility investigation of drug polymorphs giving reliable information about which form is present in solubility equilibrium. We presented an example for the possible behavior of pharmaceutical compounds.

Venlafaxine hydrochloride Form I and Form II transformed to a common product in aqueous buffer solution in $\mathrm{pH}$ 7.5-12 region and thus the measured solubility values at a given $\mathrm{pH}$ were identical. Solid isolated from $\mathrm{pH}$ 10-12 was identified as the free base, while between $\mathrm{pH}$ 7-8 the isolated common product was identified as VENL hydrochloride monohydrate based on XRPD results. Since VENL dissolves freely in acidic medium not the solubility but the better manufacturability can explain why the less stable Form II is preferably used in tablets.

We have also presented the good agreement of equilibrium solubility measured by SSF coupled with off-line analytics and in situ fiber optic concentration monitoring. $\mu$ DISS Profiler ${ }^{\mathrm{TM}}$ has the advantage to provide much more information about dissolution process, with this approach the dissolution kinetic, the supersaturation and the time needed to reach the equilibrium can be easily monitored. It is simpler and occasionally can be faster than traditional methods however there are limits of its application. Neither too high nor too low solubility can be measured with this instrument, the optimal solubility window is about $1-500 \mu \mathrm{g} / \mathrm{mL}$. Since the new APIs in drug research are mainly poorly soluble compounds, for their solubility study $\mu$ DISS Profiler seems to be advantageous.

\section{Acknowledgement}

We thank our colleague Dr. János Madarász from the Department of Inorganic and Analytical Chemistry (Budapest University of Technology and Economics) who provided insight and expertise that greatly assisted the research. This project was supported by the ÚNKP-18-3-I New National Excellence Program of the Ministry of Human Capacities, Gedeon Richter Talentum Foundation, Pro Progressio Foundation. This work was performed in the frame of FIEK_16-1-2016-0007 project, implemented with the support provided from the National Research, Development and Innovation Fund of Hungary, financed under the FIEK_16 funding scheme.

\section{References}

1. Brittain HG (editor). Polymorphism in pharmaceutical solids. 2nd edition, Marcel Dekker, 2009, New York.

2. Pangarkar PA, Tayade AM, Uttarwar SG, Wanare RS. Drug polymorphism: an overview. Int. J. Pharm. Techn. 2013;5: 2374-2402.

3. Singhal D, Curatolo W. Drug polymorphism and dosage form design: a practical perspective. Adv. Drug Deliv. Rev. 2004; 56: 335-347. https://doi.org/10.1016/j. addr.2003.10.008

4. Aguiar AJ, Krc J, Kinkel AW, Samyn JC. Effect of polymorphism on the absorption of chloramphenicol from chloramphenicol palmitate. J. Pharm. Sci. 1967; 56: 847853. https://doi.org/10.1002/jps.2600560712

5. Meyer MC, Straughn AB, Jarvi EJ, Wood GC, Pelsor FR, Shah VP. The bioinequivalence of carbamazepine tablets with a history of clinical failures. Pharm. Res. 1992; 9: 1612-1616. https://doi.org/10.1023/A:1015872626887

6. Brice GW, Hammer HF. Therapeutic nonequivalence of oxytetracycline capsules. J. Am. Med. Assoc. 1969; 208: 1189-1190. https://doi.org/10.1001/jama.208.7.1189

7. Eyjolfsson R. Enalapril maleate polymorphs: instability of form II in a tablet formulation. Pharmazie, 2002; 57: 347-348.

8. Bauer J, Spanton S, Henry R, Quick J, Dziki W, Porter W, Morris J. Ritonavir: an extraordinary example of conformational polymorphism. Pharm. Res. 2001; 18: 859-866. https://doi.org/10.1023/A:1011052932607

9. Raw AS, Furness MS, Gill DS, Adams RC, Holcombe FO, Yu LX. Regulatory considerations of 
pharmaceutical solid polymorphism in Abbreviated New Drug Applications (ANDAs). Adv. Drug Deliv. Rev. 2004; 56: 397-414. https://doi.org/10.1016/j. addr.2003.10.011

10. Saifee M, Inamdar N, Dhamecha D L, Rathi AA. Drug polymorphism: a review. Int. J. Health Res. 2009; 2: 291-306. https://doi.org/10.4314/ijhr.v2i4.55423

11. Censi R, Di Martino P. Polymorph impact on the bioavailability and stability of poorly soluble drugs. Molecules, 2015; 20: 18759-18776. https://doi.org/10.3390/ molecules201018759

12. Pudipeddi M, Serajuddin ATM. Trends in solubility of polymorphs. J. Pharm. Sci. 2005; 94: 929-939. https:// doi.org/10.1002/jps.20302

13. Llinàs A, Box KJ, Burley JC, Glen RC, Goodman JM. A new method for the reproducible generation of polymorphs: two forms of sulindac with very different solubilities. J. Appl. Cryst. 2007; 40: 379-381. https://doi. org/10.1107/S0021889807007832

14. Schinzer WC, Bergren MS, Aldrich DS, Chao RS, Dunn MJ, Jeganathan A, Madden LM. Characterization and interconversion of polymorphs of premafloxavin, a new quinolone antibiotic. J. Pharm. Sci. 1997; 86: 14261431. https://doi.org/10.1021/js970063o
15. Avdeef A, Fuguet E, Llinàs A, Ràfols C, Bosch E, Völgyi G, Verbic T, Boldyreva E, Takács-Novák K. Equilibrium solubility measurement of ionizable drugs - Consensus recommendations for improving data quality. ADMET \& DMPK, 2016; 4: 117-178. https://doi.org/10.5599/admet.4.2.292

16. Baka E, Comer J, Takács-Novák K. Study of equilibrium solubility measurement by saturation shakeflask method using hydrochlorothiazide as model compound. J. Pharm. Biomed. Anal. 2008; 46: 335-341. https://doi.org/10.1016/j.jpba.2007.10.030

17. Völgyi G, Baka E, Box K, Comer J, Takács-Novák K. Study of $\mathrm{pH}$-dependent solubility of organic bases. Revisit of Henderson-Hasselbalch relationship. Anal. Chim. Acta, 2010; 673: 40-46. https://doi.org/10.1016/j. aca.2010.05.022

18. Roy S, Bhatt PM, Nangia A, Kruger, G.J. Stable polymorph of venlafaxine hydrochloride by solid-tosolid transition at high temperature. Cryst. Growth Des. 2007; 7: 476-480. https://doi.org/10.1021/ cg0607699

19. Avdeef A. Solubility of sparingly-soluble ionisable drugs. Adv. Drug Deliv. Rev. 2007; 59: 568-590 https:// doi.org/10.1016/j.addr.2007.05.008. 